\title{
A connectionist expert system for fault diagnosis
}

\author{
Jiann-Liang Chen \\ Advanced Technology Center, Computer and Communication Laboratory, Industrial Technology Research Institute, Hsinchu \\ (Taiwan) \\ Chern-Lin Chen \\ Department of Electrical Engineering, National Taiwan University, P.O. Box 23-74, Taipei (Taiwan)
}

(Received February 18, 1992)

\begin{abstract}
The design of a connectionist expert system for the fault diagnosis of a distribution system is proposed. The heuristic rules acquired from distribution dispatchers' experiences are incorporated into the connectionist network which can be utilized as the expert system knowledge base. The data sets used to train the connectionist network are the current waveforms recorded upon the occurrence of miscellaneous faults. The interconnection weights of this network are adjusted by the back-propagation learning algorithm. The back-propagation connectionist network can then be used as an expert system for diagnosing distribution faults. A fault is identified and located by recognizing its associated current waveform. The connectionist expert system is built on a 32-bit personal computer. It is tested for diagnosing faults on a real distribution system. From the experimental results, it is found that the connectionist expert system has the following advantages: it is able to perform on-line diagnoses in a very short operation time of less than 1 second, and it makes inferences about fault types and fault locations from the locally recorded current waveforms only. This system is applicable to dispatching centres. It may assist dispatchers in judging emergency situations, which is the first step in the restoration procedure.
\end{abstract}

\section{Introduction}

The concept central to the operation of a distribution system in Taiwan Power Company is to supply power to the customers and to meet their load demands. In order to get through the work, there are two main mechanisms of a distribution system. One is to increase the capacity of the distribution system, and the other is to reduce the effect of emergency faults. Usually the latter, whether the customers are supplied with power or not, deserves more attention than the former. Hence, research on the restoration procedure is of great importance, especially the reduction of the restoration time. Fault diagnosis is the first step in restoration procedures; however, it is difficult and time consuming. In Taiwan, the overhead lines and underground cables of the distribution system are easily destroyed by external forces or insulation problems [1].

Several approaches based on switching operations and logic programming have been reported in the literature for the fault diagnosis of a dis- tribution system [2-5]. The feasibility of artificial intelligence applied to fault diagnosis has also been discussed [6-9]. However, these approaches require much computational effort and do not come up to expectation for on-line applications.

After intensive interviews with distribution dispatchers in Taiwan Power Company, it was found that current fault diagnosis practice relies on the use of the BICCOTEST Impulse Current System [10]. From the detected transient current waveform (TCW), a fault can be identified and located. The heuristic rules for fault diagnosis were developed from past experiences. The utilization of these rules in this system stimulated our development of a connectionist expert system for fault diagnosis.

Considerable progress in the application of artificial neural networks (ANNs) to power system problems has been achieved in recent years $[11,12]$. However, the application of ANNs to fault diagnosis is rather limited [13]. The purpose of this paper is to develop a diagnostic connectionist expert system, which is an expert system 
with a connectionist network (neural network) as its knowledge base. There are many types of connectionist networks. The research presented here focuses on the three-layer feed-forward network with the back-propagation learning algorithm $[14,15]$. The effectiveness of the proposed connectionist expert system is illustrated by the experimental results of diagnosing three types of faults, short-circuit, open-circuit and flashing, on a real distribution system.

The main contributions of this research are summarized as follows.

(1) A connectionist expert system is presented for the fault diagnosis of distribution systems.

(2) The heuristic rules, collected by experienced distribution dispatchers, are incorporated into the connectionist expert system.

(3) The system is able to perform on-line diagnoses in a very short operation time of less than 1 second.

(4) The system makes inferences about fault types and locations from the locally detected TCWs only.

The remaining parts of this paper are organized as follows. The concept of the connectionist expert system is introduced in $\$ 2$. The connectionist expert system for fault diagnosis is described in \$3. Experimental results on a real distribution system are given in $\$ 4$. The conclusions are presented in $\$ 5$.

\section{Connectionist expert system}

To combine a neural network with an expert system, the following questions must be considered [16].

(1) Can the learning power of neural network models be harnessed for expert system construction?

(2) Can neural network models be used for inferencing in an interactive fashion?

(3) Can connectionist expert systems be made to give reasonable justifications for their conclusions?

The above questions came up in several applications and generated positive results [17]. Hence, the design of a connectionist expert system which uses an artificial neural network for the knowledge base of an expert system is valid. The construction of a connectionist expert system is shown in Fig. 1.

The system's storage of knowledge is the interconnection strengths among processing elements, so the knowledge determines directly how

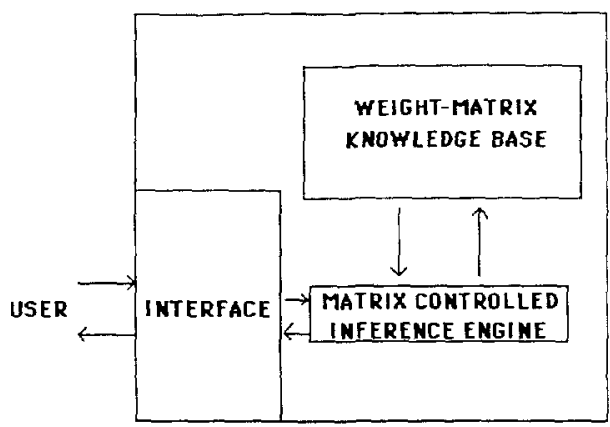

Fig. 1. 'The construction of a connectionist expert system.

the processing elements interact rather than being seated passively in a memory, waiting to be looked up by the CPU. The connectionist network generates its own knowledge by learning from the past experiences. Learning is achieved through a specified learning rule which adapts the interconnection weights among processing elements. The weight matrix of the network is thus established. The weight matrix is developed to support the inference engine, which is responsible for determination of a solution based on the knowledge base. The interface requests the inference engine to find the solution according to the user's input.

\section{Connectionist expert system for fault diagnosis}

As mentioned previously, the connectionist expert system is adopted by many researchers as the most familiar viewpoint of pattern recognition. In contrast to those approaches, we focus here on fault type identification and estimation of the fault locations in a distribution system.

\subsection{System development}

The development of the connectionist expert system for fault diagnosis is shown in Fig. 2.

There are two phases, learning and diagnosis. In the learning phase, the TCWs identified by dispatchers' experiences are fed into the system. The weight matrices are generated until the back-propagation learning algorithm converges. In the diagnosis phase, the detected TCW enters the system and the type and location of the associated fault are estimated.

\subsection{Back-propagation learning algorithm}

The back-propagation learning algorithm is an iterative gradient algorithm designed to minimize the mean square error between the actual 


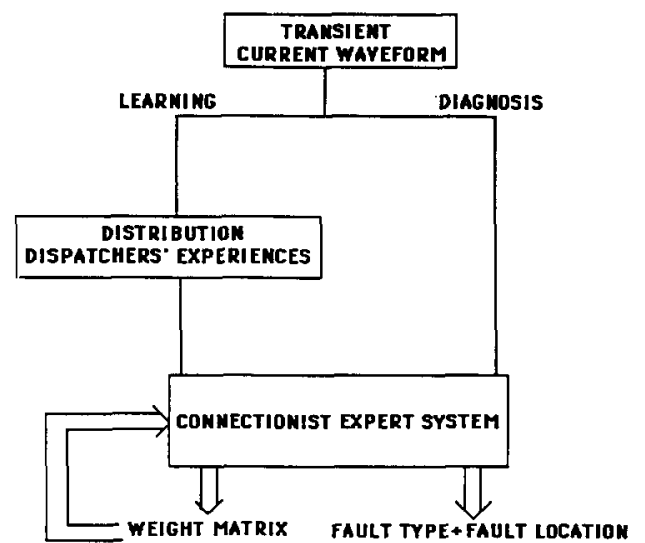

Fig. 2. The development of the connectionist expert system for fault diagnosis.

output of a multilayer feed-forward network and the desired output. An essential component of the algorithm is the iterative method that propagates error terms required to adapt weights back from nodes in the output layer to nodes in lower layers.

At the beginning, we set all weights and node offsets to small random values. The input values are presented and the desired outputs are specified. Then the network is used to calculate actual outputs. A recursive algorithm, starting at the output nodes and working back to the hidden layer, adjusts weights until they converge and the cost function is reduced to an acceptable value. The training process is repeated by presenting different sets of input data to the network.

\subsection{Fault models}

The transient behaviour of the current waveform upon the occurrence of a fault is described in this subsection.

\subsubsection{Fault type}

Three types of faults, which are usually encountered in the distribution system, and their associated TCWs are described.

(a) An open-circuit fault, that is, a conductor fault; the trace of the TCW goes up by degrees (Fig. 3(a)).

(b) A short-circuit fault, that is, one of the insulation faults; the trace is shown in Fig. 3(b).

(c) A flashing fault, that is, one of the insulation faults, like the short-circuit; Fig. 3(c) is an example of the flashing fault.

The three fault types are easily identified by the distribution dispatchers' experiences. In the connectionist expert system, fault types and their numerical representations are listed in Table 1.

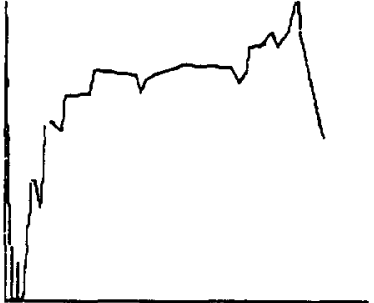

(a)

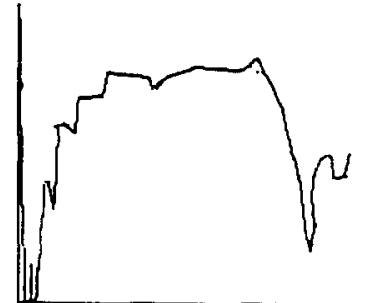

(b)

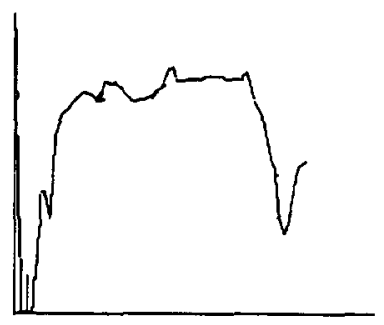

(c)

Fig. 3. The detected transient current waveforms of (a) an open-circuit fault, (b) a short-circuit fault and (c) a flashing fault.

TABLE 1. Fault types and their numerical representations

\begin{tabular}{ll}
\hline Fault type & Numerical range \\
\hline Open-circuit & $0.5-1.5$ \\
Short-circuit & $1.5-2.5$ \\
Flashing & $2.5-3.5$ \\
\hline
\end{tabular}

\subsubsection{Fault location}

In the TCW, the elapsed time between the first two impulses is proportional to the electrical distance from the fault location to the detector. Experienced dispatchers can estimate the fault point by this distinction. Only fault locations of $1200 \mathrm{~m}$ which were obtained from the dispatchers' knowledge are shown in Fig. 3.

\section{Test study}

\subsection{Study system}

The system under study is a distribution system in the northern area of Taipei. There are one substation and ten underground cables which are composed of cross-linked polyethylene in this study system. The voltage level of each undergound cable is $11.4 \mathrm{kV}$ and the faults are indicated by I, II and III. The TCW of each fault is described in Fig. 4.

\subsection{Simulation results}

The developed connectionist expert system is shown in Fig. 5. The input data are sampled from the detected TCW. The output layer gives the 


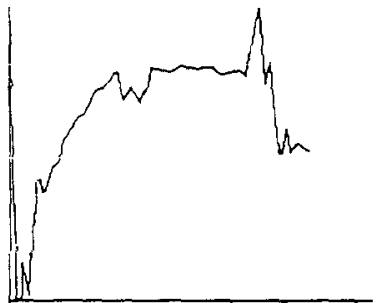

(a)

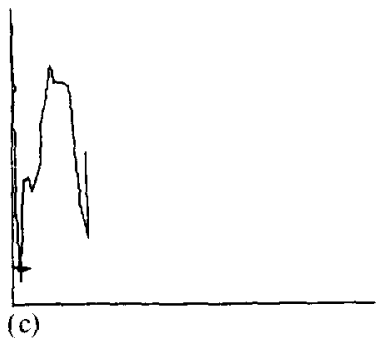

Fig. 4. The detected transient current waveforms of (a) fault I, (b) fault II and (c) fault III.

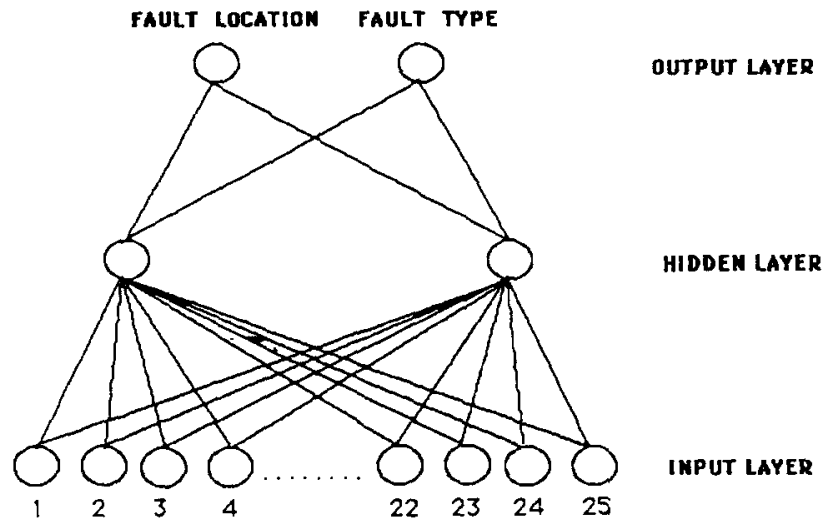

Fig. 5. The developed connectionist expert system.

fault type and the distance of the fault location. The network contains 25 nodes in the input layer, 2 nodes in the hidden layer, and 2 nodes in the output layer. The weight matrices which contain the input-hidden layer and the hidden-output layer of the connectionist expert system are shown in Fig. 6. The fault locations and fault types of the three fault points, which are indicated by I, II and III in Fig. 4 are given in Table 2 . The results computed by the connectionist

$\left[\begin{array}{rr}-0.0759 & -0.4200 \\ 0.0367 & 0.7106 \\ 0.7378 & 0.8254 \\ 0.2416 & -0.5517 \\ 0.5989 & 1.3808 \\ 0.0306 & -10.9078 \\ 0.2862 & 4.4282 \\ 0.0042 & 2.3256 \\ -1.1746 & -1.0736 \\ 0.0499 & 0.6489 \\ -1.4358 & -2.1105 \\ 0.0012 & 2.3998 \\ -0.9776 & -0.5972 \\ 0.4798 & -1.1030 \\ -0.9594 & -0.5790 \\ 0.4600 & -1.1228 \\ 0.0021 & 2.3619 \\ -0.1057 & -0.1469 \\ 0.2084 & 1.3756 \\ -0.0162 & 2.2563 \\ -0.5681 & 0.0968 \\ -1.2890 & -1.1361 \\ -2.4530 & -5.0387 \\ -4.2477 & -1.8848 \\ -2.9463 & 5.5498\end{array}\right]$

(a)

$\left[\begin{array}{rr}-2.8315 & 0.0000 \\ 0.0000 & -8.6536\end{array}\right]$

(b)

Fig. 6. The weight matrices: (a) input-hidden; (b) hidden output.

expert system are compared with the actual values. Their deviations are also listed in the Table.

\subsection{Discussion}

From the presented results, several observations are in order.

(1) As observed from Table 2, the deviations between the values of fault locations computed by the proposed approach and actual values are all below $1.5 \%$. Very accurate estimates are formed by the developed connectionist expert system.

TABLE 2. The simulation results of fault locations and types

\begin{tabular}{lllllll}
\hline Fault point & $\begin{array}{l}\text { Fault location } \\
(\mathrm{m})\end{array}$ & $\begin{array}{l}\text { Actual value } \\
(\mathrm{m})\end{array}$ & $\begin{array}{l}\text { Error } \\
(\mathrm{m})\end{array}$ & $\begin{array}{l}\text { Deviation } \\
(\%)\end{array}$ & $\begin{array}{l}\text { Fault-type } \\
\text { output }\end{array}$ & $\begin{array}{l}\text { Indicated } \\
\text { fault type }\end{array}$ \\
\hline I & 1179 & 1168 & 11 & 0.94 & 0.96 & Open-circuit \\
II & 1108 & 1094 & 14 & 1.30 & 1.94 & Short-circuit \\
III & 256 & 256 & 0 & 0.00 & 2.77 & Flashing \\
\hline
\end{tabular}


(2) The numerical value of the fault-type output indicates which kind of fault it is close to. In our tests, the fault-type output generated the correct types of these three kinds of fault.

(3) The required CPU time on a 32-bit personal computer is less than one second. Hence, the system is able to perform on-line distribution fault diagnosis.

\section{Conclusions}

A connectionist expert system has been developed for the fault diagnosis of a distribution system in Taiwan. The system is implemented on a 32-bit personal computer. A feed-forward threelayer network with a back-propagation learning algorithm is described and constructed. This network contains 25 nodes in the input layer, 2 nodes in the hidden layer, and 2 nodes in the output layer. The proposed connectionist expert system approach was tested on a distribution system in the northern area of Taiwan. The locations and types of three different faults were estimated. The results are satisfactory and demonstrate well the capability of the developed connectionist expert system. With sufficient learning from past experiences, that is, the TWCs, the distances of fault locations were computed with tiny deviations, all below $1.5 \%$, from the actual values. The required computing time was less than one second. In view of the successful experiments, it is the authors' belief that the connectionist expert system will be a valuable tool to assist distribution dispatchers in on-line fault diagnosis.

\section{References}

1 Y. Y. Hsu, J. L. Chen and L. M. Chen, Development of a software program for the evaluation of distribution system reliability, Res. Rep., Taiwan Power Company, June 1988.
2 C. H. Castro, J. B. Bunch and T. M. Topka, Generalized algorithms for distribution feeder deployment and sectionalizing, IEEE Trans., PAS-99 (1980) 549-557.

$3 \mathrm{~K}$. P. Wong and C. P. Tsang, A logic programming approach to fault diagnosis in distribution ring networks, Electr. Power Syst. Res., 15 (1988) 77-87.

4 M. B. Guertin and Y. Lamarre, Reliability analysis of substations with automatic modelling of switching operations, IEEE Trans., PAS-94 (1975) 1599-1605.

5 B. M. Aucoin and B. D. Russell, Distribution high impedance fault detection utilizing high frequency current components, IEEE Trans., PAS-101 (1982) 1596-1606.

6 C. Fukui and J. Kawakami, An expert system for fault section estimation using information from protective relays and circuit breakers, IEEE Trans., PWRD-1 (4) (1986) 83-90.

7 T. Sakaguchi and K. Matsumoto, Development of a knowl. edge based system for power system restoration, IEEE Trans., PAS-102 (1983) 320-329.

8 P. K. Kalra, Fault diagnosis for an HVDC system: a feasibility study of an expert system application, Electr. Power Syst. Res., 14 (1988) 83-89.

9 S. N. Talukdar, E. Cardozo and T. Perry, The operator's assistant-an intelligent, expandable program for power system trouble analysis, IEEE Trans., PWRS-1 (1986) 182187.

10 P. F. Gale, The application of digital electronics to power cable fault location and testing, BICCOTEST Instruments, 1980.

11 D. J. Sobajic and Y. H. Pao, Artificial neural-nets based dynamic security assessment for electric power system, IEEE Trans., PWRS-4 (1989) 220-228.

12 N. I. Santoso and O. T. Tan, Neural-net based real-time control of capacitors installed on distribution systems, IEEE Trans., PWRD-5 (1990) 266-272.

13 S. Ebron, D. L. Lubkeman and M. White, A neural network approach to the detection of incipient faults on power distribution feeder, IEEE Trans., PWRD-5 (2) (1990) 905914.

14 D. E. Rumelhart, G. E. Hinton and R. J. Williams, Learning internal representations by error propagation: parallel distributed processing, Explorations in the Microstructures of Cognition, Vol. 1, MIT Press, Cambridge, MA, 1986, pp. 318-362.

15 D. E. Rumelhart, G. E. Hinton and R. J. Williams, Learning representations by back-propagating errors, Nature (London), 323 (1986) 696-699.

16 S. I. Gallant, Connectionist expert system, Commun. ACM, 2 (1988) $152-169$.

17 G. E. Hinton and J. A. Anderson, Parallel Models of Associative Memory, Erbaum, Hillsdale, NJ, 1981. 